\title{
Article \\ Study of Microwave-Induced Ag Nanowire Welding for Soft Electrode Conductivity Enhancement
}

\author{
Meng Zhang ${ }^{1,+}$, Songjia Han ${ }^{2,+}{ }^{,}$Zhi-Yang Xuan ${ }^{2}$, Xiaohui Fang ${ }^{3}$, Xiaoming Liu ${ }^{4, * \mathbb{C}}$, Wu Zhang ${ }^{3, *}$ \\ and Hui-Jiuan Chen ${ }^{2, *}$ \\ 1 Precision Medicine Institute, The First Affiliated Hospital of Sun Yat-Sen University, Sun Yat-Sen University, \\ Guangzhou 510080, China; meng.zhang_china@outlook.com \\ 2 State Key Laboratory of Optoelectronic Materials and Technologies, \\ School of Electronics and Information Technology, Sun Yat-Sen University, Guangzhou 510006, China; \\ hansongiia@126.com (S.H.); ZYang_Xuan@outlook.com (Z.-Y.X.) \\ 3 College of Physical and Material Engineering, Guangzhou University, Guangzhou 510006, China; \\ fangxiaohui@gzhu.edu.cn \\ 4 College of Physics and Electronic Information, Anhui Normal University, Wuhu 241003, China \\ * Correspondence: xiaoming.liu@ahnu.edu.cn (X.L.); zh0002wu@outlook.com (W.Z.); \\ chenhuix5@mail.sysu.edu.cn (H.-J.C.) \\ + The two authors contributed equally to this paper.
}

Citation: Zhang, M.; Han, S.; Xuan, Z.-Y.; Fang, X.; Liu, X.; Zhang, W.;

Chen, H.-J. Study of

Microwave-Induced Ag Nanowire Welding for Soft Electrode Conductivity Enhancement. Micromachines 2021, 12, 618. https:// doi.org/10.3390/mi12060618

Academic Editor: Atanas Ivanov

Received: 5 April 2021

Accepted: 25 May 2021

Published: 27 May 2021

Publisher's Note: MDPI stays neutral with regard to jurisdictional claims in published maps and institutional affiliations.

Copyright: (c) 2021 by the authors. Licensee MDPI, Basel, Switzerland. This article is an open access article distributed under the terms and conditions of the Creative Commons Attribution (CC BY) license (https:/ / creativecommons.org/licenses/by/ $4.0 /)$.

\begin{abstract}
Silver nanowire (AgNW)-coated thin films are widely proposed for soft electronics application due to their good conductivity, transparency and flexibility. Here, we studied the microwave welding of AgNW-based soft electrodes for conductivity enhancement. The thermal effect of the microwave to AgNWs was analyzed by dispersing the nanowires in a nonpolar solution, the temperature of which was found to be proportional with the nanowire diameters. AgNWs were then coated on a thin film and welded under microwave heating, which achieved a film conductivity enhancement of as much as 79\%. A microwave overheating of AgNWs, however, fused and broke the nanowires, which increased the film resistance significantly. A soft electrode was finally demonstrated using the microwave-welded AgNW thin film, and a $1.13 \mu \mathrm{A} / \mathrm{mM}$ sensitivity was obtained for glucose sensing. Above all, we analyzed the microwave thermal effect on AgNWs to provide a guidance to control the nanowire welding effect, which can be used for film conductivity enhancement and applied for soft and bio-compatible electrodes.
\end{abstract}

Keywords: Ag nanowires; microwave heating; thermal welding; glucose sensing

\section{Introduction}

Electrodes with good conductivity, conformability, and bio-compatibility are highly desired in soft electronics for signal sensing, motion detection and health care applications [1]. In early years, indium tin oxide (ITO) was used for soft electrodes due to its good transparency and conductivity. Nonetheless, ITO fabrication requires special material growth, high deposition temperature and vacuum processing [2]. In addition, ITO is also easy to crack when getting bent, which largely limits its applications in the fast-growing wearable electronic technology. Various other materials were later proposed to substitute ITO, such as conductive polymers, nanotubes and metallic nanowires, etc. [3-8]. However, polymer usually has the limitation of low conductivity, while the nanotubes could be undermined due to tube defects or bundling. Metallic nanowires (MNWs), instead, have the advantages of good flexibility, high transparency, large conductivity and cost effectiveness. Meanwhile, MNWs can be easily coated on soft thin films such as poly(ethylene terephthalate) (PET) or polydimethylsiloxane (PDMS), therefore they have become a promising candidate for soft electrodes in wearable technology [9-12].

When coated on thin films, the MNWs are physically contacted with each other and they form a network texture. The contact resistance between MNWs will inevitably affect 
the film electrical performance. Many remarkable works have been carried out to reduce the MNW contact resistance, including cold welding [6], plasmonic welding [13-15], laser sintering [16], mechanical pressure [17], thermal annealing [18,19], electron-beam-induced welding [20], solvent-induced welding [21,22] and so on. The above works can be generally categorized into pressure welding and heat welding approaches. The pressure welding approach loads large pressure on the whole device without selectivity, which is energy consuming and may lead to thin film damage. The traditional heat welding approach, instead, requires very delicate and expensive equipment to control thermal effect on a small selected area, which increases the cost and prevents large scale fabrication. Therefore, a simple welding method that satisfies both selective processing and large scale fabrication is highly demanded.

Microwave heating is a widely used method for the synthesis of nano-materials because of its advantages in thermal effect selectivity, reduced processing time and enhanced product purity $[23,24]$. Recently, some scientists proposed employing microwave treatment for MNW welding, which significantly improved the thermal and electrical conductivity of the nanowire-coated films [25]. While these preliminary works only focused on the demonstration of microwave welding of MNWs, a quantitative analysis of the microwave thermal effect on the MNWs is necessary for guiding the microwave welding control in real applications. In this work, we analyzed the microwave-induced temperature increase, welding and the conductivity enhancement of AgNWs at different nanowire densities, diameters and different microwave power and time applied. The conductivity enhancement was optimized for the AgNW-coated thin films, which was applied as a soft electrode for glucose concentration sensing.

\section{Materials}

Below are the materials for the AgNWs synthesis and experiment process: silver nitrate $\left(\mathrm{AgNO}_{3}, \mathrm{CAS}: 7761-88-8,99.9999 \%\right.$, Sigma-Aldrich, St. Louis, MI, USA), cupric chloride $\left(\mathrm{CuCl}_{2}, \mathrm{CAS}: 7447-39-4,99 \%\right.$, Sigma-Aldrich, St. Louis, MI, USA), ethylene glycol (EG, CAS: 107-21-1, anhydrous 99.8\%, Sigma-Aldrich), polyvinyl pyrrolidone (PVP, CAS: 9003-39-8, MW = 360,000, Sigma-Aldrich), polydimethylsiloxane (PDMS, DC Sylgard 184), polyethylene terephthalate (PET, Dupont, Shenzhen, China). Before synthesis, $\mathrm{AgNO}_{3}$, $\mathrm{CuCl}_{2}$, and PVP were dissolved in EG to form a uniform solution.

\section{Methods}

\subsection{Synthesis of AgNWs}

The AgNWs were synthesized through the reduction of a metal salt precursor by a polyol. First, $20 \mathrm{~mL}$ ethylene glycol (EG) was heated to $155^{\circ} \mathrm{C}$ to remove all water inside. Then, $160 \mu \mathrm{L}(4 \mathrm{mM}) \mathrm{CuCl}_{2}$ solution and $6 \mathrm{~mL}(115 \mathrm{mM})$ polyvinyl pyrrolidone (PVP) solution were successively added into the EG with a 15 min interval time. Finally, $6 \mathrm{~mL}$ $(95 \mathrm{mM}) \mathrm{AgNO}_{3}$ solution was added to the mixture, which was maintained at $155^{\circ} \mathrm{C}$ for two hours to ensure a full synthesis of AgNWs. The AgNWs were then rinsed several times using acetone and deionized (DI) water for purification. The purified AgNWs were then dispersed in ethanol for next step experiments.

\subsection{AgNWs Dispersion in Nonpolar Solution}

AgNWs were dispersed into a base solution to evaluate the microwave thermal effect on the nanowires. Nonpolar liquid Isopar $\mathrm{H}$ was considered as the base solution, which has a low dielectric constant of 1.89 and high boiling point of $189^{\circ} \mathrm{C}$. Due to its non-polarity, Isopar $\mathrm{H}$ absorbs very little microwave energy and therefore will not affect the microwave heating analysis on AgNWs. AgNWs, however, are hydrophilic and will aggregate in Isopar $\mathrm{H}$ solution (Figure 1a). We therefore modified the base solution recipe by adding $\mathrm{CH}$ 5 hyperdispersant ( $5 \mathrm{wt} \%$ ) and Span-20 surfactant (10 wt\%) to the Isopar $\mathrm{H}$. The AgNWs in ethanol from Section 3.1 were centrifuged at $5000 \mathrm{rpm}$ for $5 \mathrm{~min}$, and then heated at $70{ }^{\circ} \mathrm{C}$ for $10 \mathrm{~min}$ to remove the residual ethanol. AgNWs free of ethanol were quickly dumped 
into the base solution and agitated strongly with vortex mixer for $5 \mathrm{~min}$. Homogenous AgNW dispersion was obtained, as shown Figure 1b. Microwave radiation was applied to the solution at a frequency of $2.45 \mathrm{GHz}$. The solution temperature was continuously monitored through a sensor system (OMEGA FOB-100 series) (Figure 1c) for quantitatively analyzing the microwave thermal effect on AgNWs.
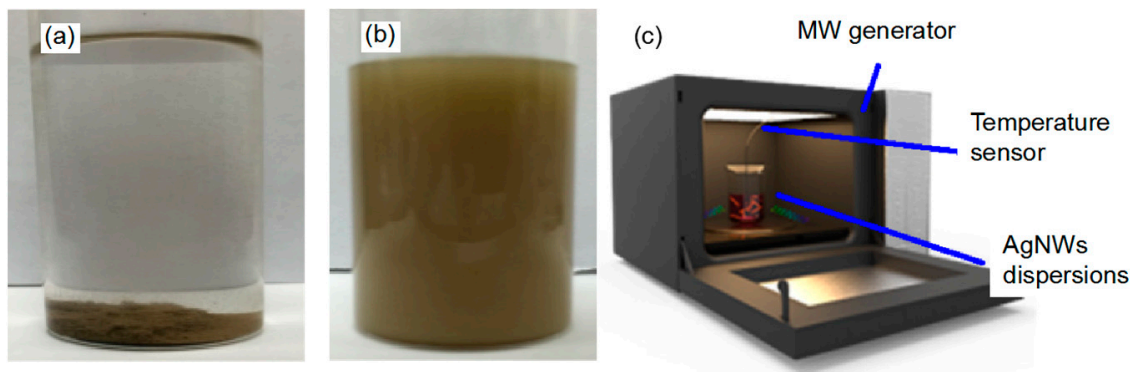

Figure 1. (a) AgNWs aggregation in Isopar H solution; (b) AgNWs dispersion in base solution of Isopar $\mathrm{H}$, Span-20 and $\mathrm{CH}-5$; (c) the setup for base solution heated by microwave.

\subsection{AgNW Coated on PET Films}

The conductivity of AgNW-coated thin films can be enhanced by the microwave welding of nanowires. Here, a PET film was used as the nanowire substrate for its good transparency and softness, which is suitable for soft electronic applications. The synthesized nanowires were first dispersed uniformly in ethanol at weight concentration $0.0125 \mathrm{wt} \%$, and then dropped along one edge of the PET film. A Meyer rod was used to roll the solutions across the film, as shown in Figure 2. Multiple layers of AgNWs can be obtained by repeating the above steps multiple times, while the rolling direction was switched to the orthogonal one after each step to ensure better uniformity. The coated film was heated at $70{ }^{\circ} \mathrm{C}$ for $1 \mathrm{~min}$ to remove the residue ethanol.

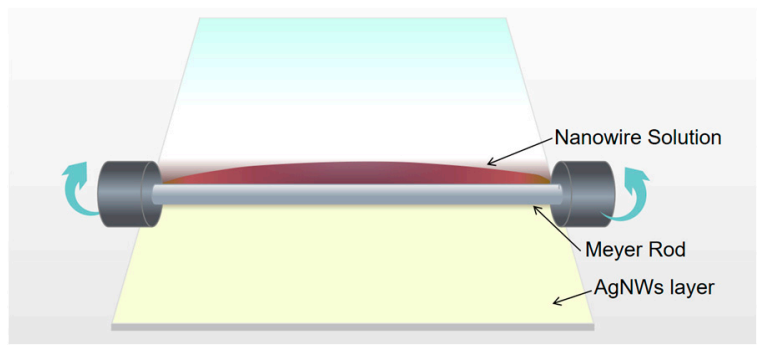

Figure 2. AgNW coated on PET films using Meyer rod.

\subsection{AgNWs Coated on PDMS for Electrode Application}

We coated AgNWs on a PDMS film as a soft and bio-compatible electrode for biosensor application. The electrode was formed by first creating a hydrophilic area on the film using oxygen plasma treatment through a shadow mask, as shown in Figure 3. The AgNWs ethanol solution with nanowire diameter of $90 \mathrm{~nm}$ and concentration of $10 \mathrm{mg} / \mathrm{mL}$ was then dropped on the film. AgNWs adhered well on the hydrophilic area and formed the electrode pattern after the ethanol volatilized. The microwave was applied to the AgNWs for $30 \mathrm{~s}$ to improve the electrode conductivity. Glucose oxidase $\left(\mathrm{GO}_{\mathrm{x}}\right)$ was then dropped on the electrode for electrochemical sensing of glucose. 


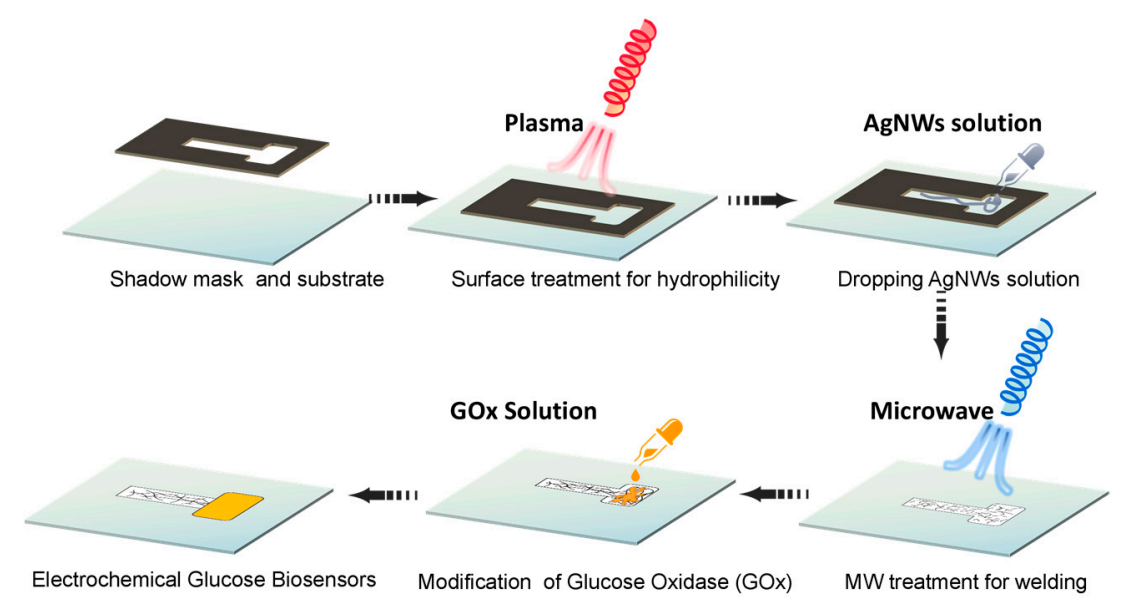

Figure 3. AgNW-coated PDMS electrode fabrication process.

\subsection{Cell Seeding for AgNW Bio-Compatibility Testing}

We seeded T24 cells on four AgNW-coated PDMS thin films, which were then placed in a 48-well plate for AgNW bio-compatibility testing. On each film, about 20,000 cells in $100 \mu \mathrm{L}$ completed medium were seeded, and then cultured in an incubator at $37^{\circ} \mathrm{C}$ temperature and $5 \% \mathrm{CO}_{2}$ concentration. The cell growth on the four culturing groups was investigated after 12, 24, 48 and $72 \mathrm{~h}$, respectively. Meanwhile, the same amount of T24 cells were cultured directly in a fifth well for $72 \mathrm{~h}$ as a control group, while keeping other parameters the same. For cell growth investigation, $5 \mu \mathrm{M}$ fluorescent dye of Calcein-AM (ThermoFisher C3100MP, Waltham, MA, USA) was used.

\section{Results and Discussion}

\subsection{Structure Investigation of Synthesized AgNWs}

The synthesized AgNWs in Section 3.1 were coated on a thin PET film for structure investigation using an electronic scanning microscope (SEM, Hitachi S-4800) as shown in Figure $4 \mathrm{a}, \mathrm{b}$. The AgNWs formed a network structure on the thin film with a diameter if about $30 \mathrm{~nm}$. The diameter of AgNWs can be adjusted by altering the amount of $\mathrm{CuCl}_{2}$ in the synthesis process. As shown in Figure $4 \mathrm{c}, \mathrm{d}$, the average diameter of AgNWs was increased to $90 \mathrm{~nm}$ and $120 \mathrm{~nm}$ when the added volume of the $\mathrm{CuCl}_{2}(4 \mathrm{mM})$ was increased to $320 \mu \mathrm{L}$ and $480 \mu \mathrm{L}$ in the synthesis process, respectively. Figure $4 \mathrm{e}$ shows the average diameters of the nanowires in different deviation values.
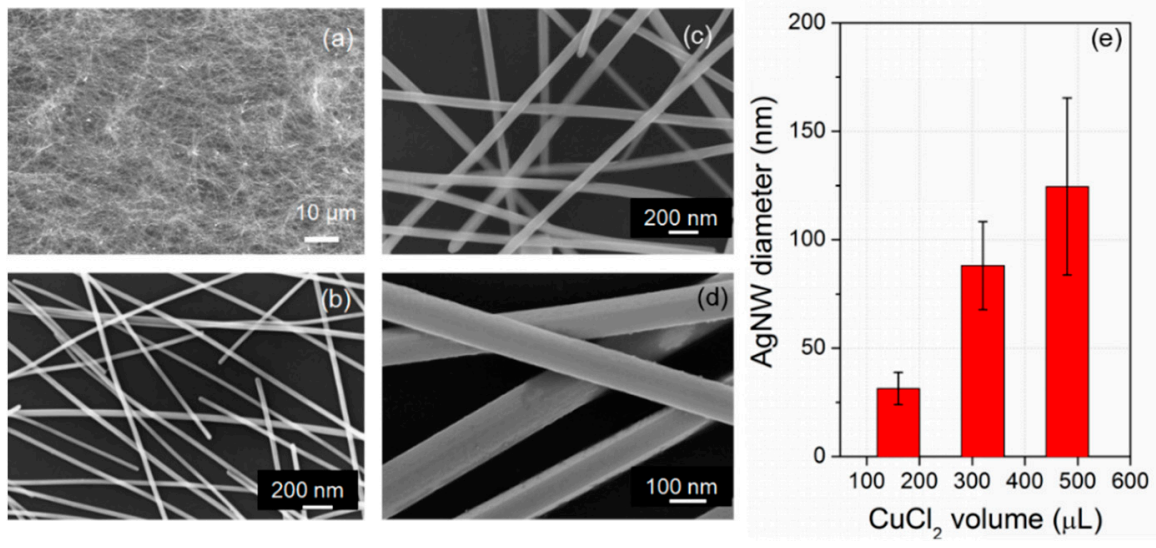

Figure 4. (a) AgNWs network; (b) close-up image of AgNWs with average diameter of $30 \mathrm{~nm}$; (c) average diameter of $90 \mathrm{~nm}$; and (d) average diameter of $120 \mathrm{~nm}$. (e) The average diameter of AgNWs at different added $\mathrm{CuCl}_{2}$ volume in the process. 


\subsection{Microwave Heating on AgNWs in Nonpolar Solution}

To understand the microwave thermal effect on the AgNWs, we monitored the temperature of AgNWs dispersed nonpolar solution under microwave heating. Firstly, a $300 \mathrm{~W}$ microwave was applied to $20 \mathrm{~mL}$ Isopar $\mathrm{H}$ base solution (without AgNWs). For comparison, the temperature of the same volume of DI water under the same microwave radiation was measured. The initial temperature was set at room temperature, $25^{\circ} \mathrm{C}$. As shown in Figure 5, the temperature increase $\Delta T$, was measured as low as $0.78 \mathrm{~K}$ for the base solution after a total $30 \mathrm{~s} \mathrm{time} \mathrm{microwave} \mathrm{heating,} \mathrm{which,} \mathrm{on} \mathrm{the} \mathrm{other} \mathrm{hand,} \mathrm{was} \mathrm{almost}$ $20 \mathrm{~K}$ for DI water. This confirmed that the base solution absorbs little microwave energy compared to conventional polar solvent.

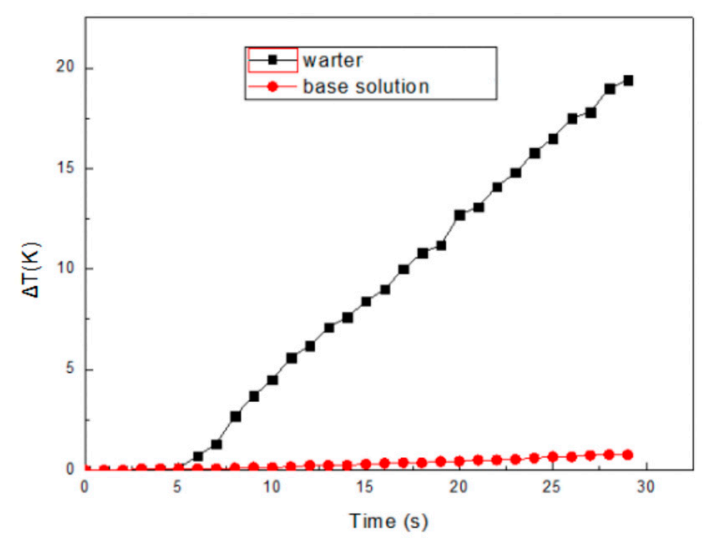

Figure 5. Temperature increase comparison for base solution and water under microwave heating.

We then monitored the temperature increase in the $20 \mathrm{~mL}$ base solution when dispersing different amounts of AgNWs in the solution. The initial temperature was kept at $25^{\circ} \mathrm{C}$. As microwave penetration depth of $\mathrm{Ag}(\approx 1.3 \mu \mathrm{m})$ [26] is much higher than the AgNW diameter, a strong electrical current in AgNWs is excited by the electrical and magnetic field of the microwave and a large amount of heat is generated [27]. Under $300 \mathrm{~W}$ microwave heating, the solution temperature increased by $1.22,2.07$ and $3.73 \mathrm{~K}$ after $30 \mathrm{~s}$ for the base solution with AgNW weight concentration $C_{\mathrm{wt}}=0.0125 \%, 0.025 \%$ and $0.05 \%$, respectively, as shown in Figure 6a. Considering the temperature increase in the base solution was $0.78 \mathrm{~K}$, the additional temperature increase due to AgNWs was $0.44,1.29$ and $2.95 \mathrm{~K}$ for the above three concentration solutions, respectively. This indicated a proportional relation between the temperature increase and the AgNW weight concentration.
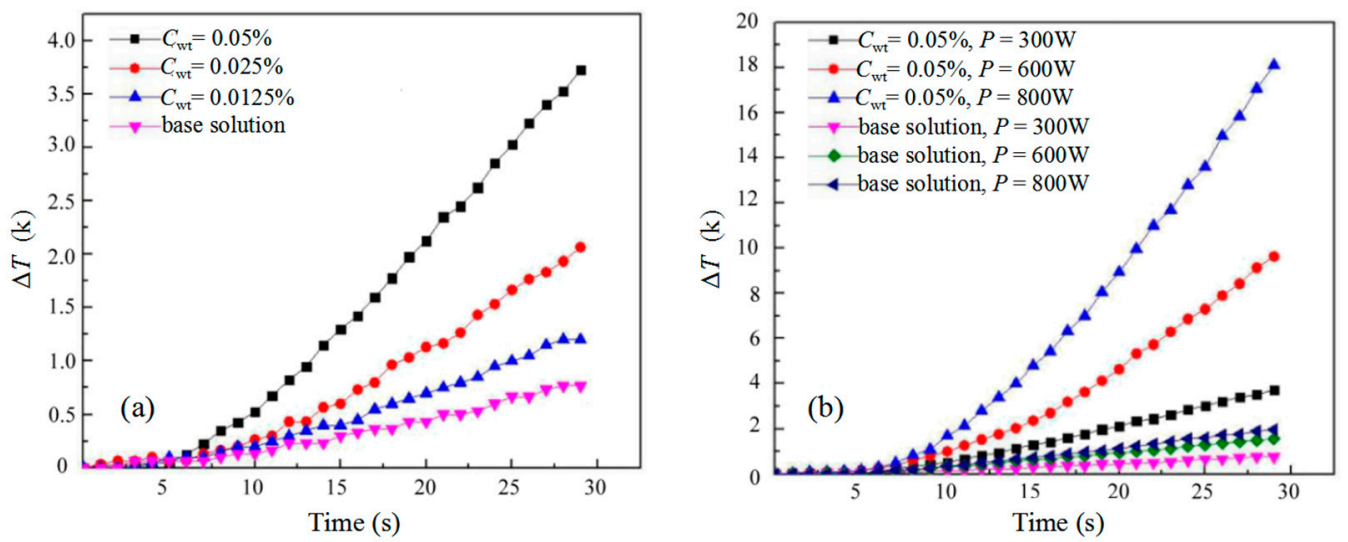

Figure 6. (a) Temperature change of AgNWs in the nonpolar base solution at various concentrations; (b) temperature change of AgNWs in the nonpolar base solution under different microwave powers. 
To further analyze the microwave heating on AgNWs, we tested the thermal effect of $20 \mathrm{~mL}$ base solution without/with $0.05 \mathrm{wt} \%$ AgNWs under different microwave heating powers. After $30 \mathrm{~s}$ microwave heating, the temperature increased by $0.78,1.54$ and $1.99 \mathrm{~K}$ for solution without AgNWs, and by 3.73, 9.62 and $18.14 \mathrm{~K}$ for solution with AgNWs at microwave power of $300 \mathrm{~W}, 600 \mathrm{~W}$ and $800 \mathrm{~W}$, respectively, as shown in Figure 6b. Therefore, the temperature increases due to AgNWs were 2.95, 8.08 and $16.15 \mathrm{~K}$ at microwave power of $300 \mathrm{~W}, 600 \mathrm{~W}$ and $800 \mathrm{~W}$, respectively. It can be seen that the temperature change depended on the microwave power linearly. It is also worth noting that when the power increased from $300 \mathrm{~W}$ to $600 \mathrm{~W}$ and then to $800 \mathrm{~W}$, the temperature of the base solution only increased by $97 \%$ and $155 \%$, while the temperature increase due to AgNWs was $212 \%$ and $447 \%$. This, again, demonstrated a high microwave power absorption ability of AgNWs. We then compared microwave heating on AgNWs of different diameters $d=30 \mathrm{~nm}, 90 \mathrm{~nm}$ and $120 \mathrm{~nm}$ in the $20 \mathrm{~mL}$ base solution at a fixed weight concentration of $0.05 \mathrm{wt} \%$. The temperature increase of solution with different diameter AgNWs was recorded every $5 \mathrm{~s}$, as shown in Figure 7, which was 2.3, 4.8 and $7.8 \mathrm{~K}$ for base solution with AgNWs in diameter of 30, 90 and $120 \mathrm{~nm}$, respectively, indicating a linear relation of the microwave thermal effect with the nanowire diameter.

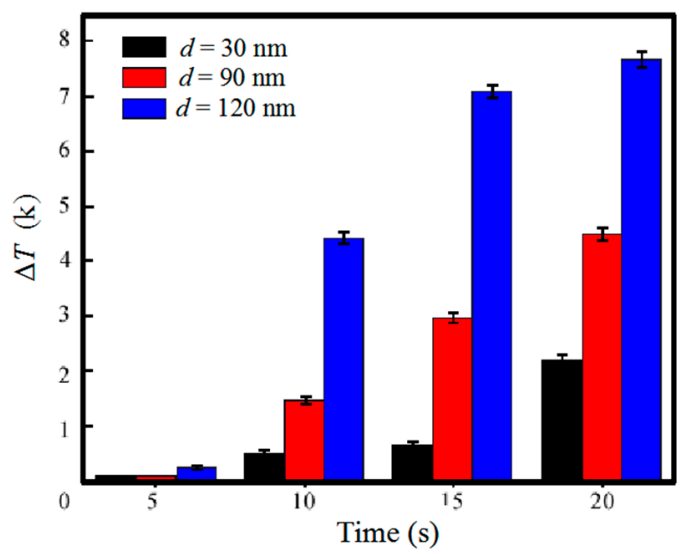

Figure 7. Temperature change of AgNWs dispersion at the power of $300 \mathrm{~W}$ with the concentration of $0.05 \mathrm{wt} \%$ for AgNWs with different diameters of 30, 90 and $120 \mathrm{~nm}$.

\subsection{Microwave Heating of AgNWs on PET Thin Film}

In this section we investigated the microwave welding of the AgNWs coated on a thin PET film, as shown in Figure 8a. The film was coated with two layers of AgNWs and manifested excellent transparency. A contacted network was observed under optical microscope, as shown in Figure $8 \mathrm{~b}$. The optical transmissions reached about $80 \%$ in the whole visible spectrum range for films with AgNW diameter of $30 \mathrm{~nm}, 90 \mathrm{~nm}$ and $120 \mathrm{~nm}$, respectively, as shown in Figure 8c. The slight drop in the transmission for larger diameter AgNW film is resulted from the slightly higher light reflection by the larger AgNWs.

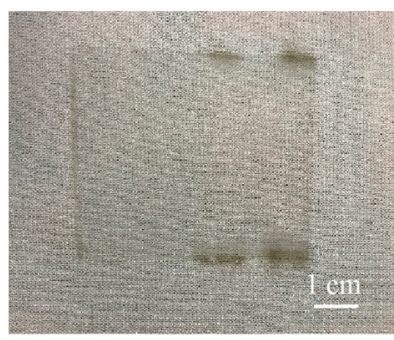

(a)

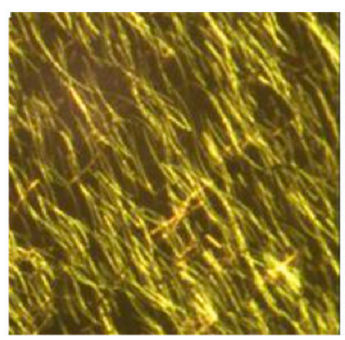

(b)

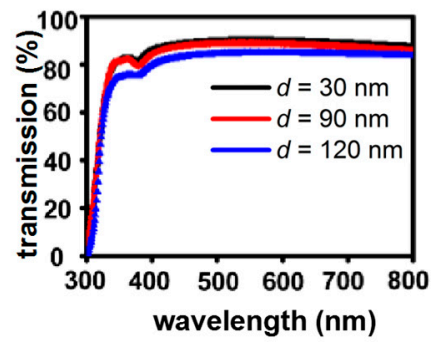

(c)

Figure 8. (a) Photograph of two layer AgNW-coated PET film; (b) nanowire structure under optical microscope; (c) optical transmittance of AgNWs film. 
The microwave welding effect of AgNWs on thin PET film was then evaluated using nanowires with diameter of $90 \mathrm{~nm}$, which have a better transmittance than that of $120 \mathrm{~nm}$ AgNWs, and a stronger thermal effect than $30 \mathrm{~nm}$ AgNWs. The SEM image of the AgNW network is shown in Figure 9(a1) before microwave heating. The AgNWs randomly distributed on the film and contacted with each other as shown in Figure 9(a2,a3). The electrical resistance at the contacted junctions, namely contact resistance, was significantly higher than that of the nanowire itself, and could induce local hotspots by microwave heating [28]. After $30 \mathrm{~s}$ microwave heating, we observed that AgNWs started to weld together at some contact junctions (Figure 9(b3)), while remaining loosely contacted at the rest (Figure 9(b2)). This indicates that the heating was not uniform at all junctions. As the microwave heating continued to $90 \mathrm{~s}$, most contact junctions were welded. In addition, the thermal effect was so large that some AgNWs started to melt (Figure 9(c2)) and even fuse, as shown in Figure 9(c3). The fused points were mostly closed to the contact junctions instead of at the contact junctions, implying that the heat generated was not enough to fuse welding junctions, but can only be conducted to nearby locations and fuse the nanowire.

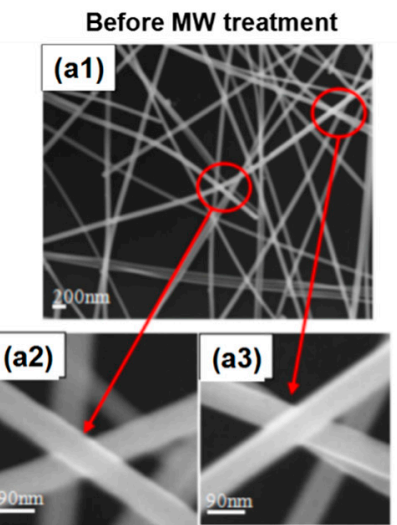

(a)

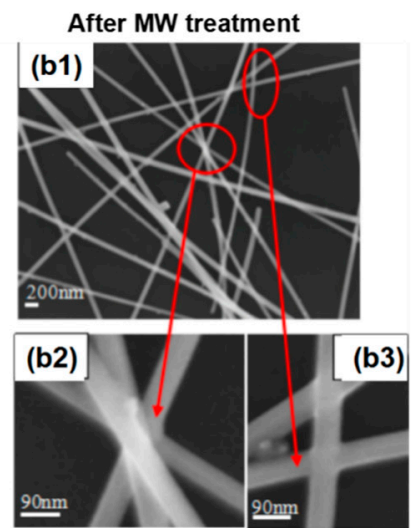

(b)

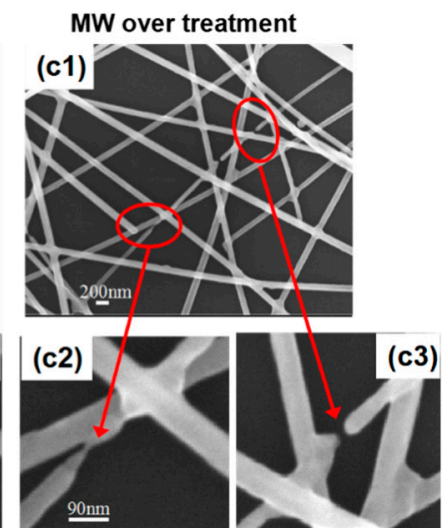

(c)

Figure 9. (a) AgNWs junction before microwave heating; (b) AgNWs junction after microwave heating; (c) AgNWs junction by an over-treatment of microwave.

The impact of microwave heating on the electrical resistance change of AgNW-coated thin film was investigated. The $90 \mathrm{~nm}$ diameter AgNWs of 1-5 layers were coated on five PET thin films, respectively, for resistance comparison. The thin film was heated by microwave radiation with power of $300 \mathrm{~W}$ at a frequency of $2.4 \mathrm{GHz}$, and the resistance changes were recorded every $30 \mathrm{~s}$, as plotted in Figure 10. Before microwave treatment, the initial film resistance $R_{0}$ was $2370,658,385,234$ and $97 \Omega / \mathrm{m}^{2}$ for films with 1, 2, 3, 4 and 5 layers of AgNWs, respectively, as indicated at the $0 \mathrm{~s}$ time in Figure 10a-e. In the first $30 \mathrm{~s}$, the resistance started to decrease for all five thin film cases, which was due to the welding of the contacted junctions in the AgNW network and agreed with the SEM image as illustrated in Figure 9b. As the microwave irradiation time increased from $30 \mathrm{~s}$ to $60 \mathrm{~s}$, the resistances for thin films with AgNWs of 1 layer and 5 layers continued to decrease due to the welding effect, while for thin films coated with 2, 3, and 4 layers of AgNWs, the resistance started to increase due to the fuse of the AgNWs, corresponding to the case in Figure 9c. After $60 \mathrm{~s}$ treatment, the nanowires were over heated by the microwave, and the nanowire fuse increased the electrical resistance significantly, which was even about 10 times larger than the original value for the 1 layer coated film under $90 \mathrm{~s}$ treatment. The relative electrical resistances $R_{\mathrm{T}} / R_{0}$ for different AgNWs layered films are plotted in Figure $10 \mathrm{f}$ for comparison. It can be seen that the electrical resistance for films with 1 to 5 layers can be decreased by as much as $79 \%, 52 \%, 67 \%, 25 \%$ and $37 \%$ after the microwave treatment for $60,30,30,30$ and 60 s correspondingly. This indicates that the microwave welding time needs to be controlled to realize an optimized conductivity enhancement for the thin film. 

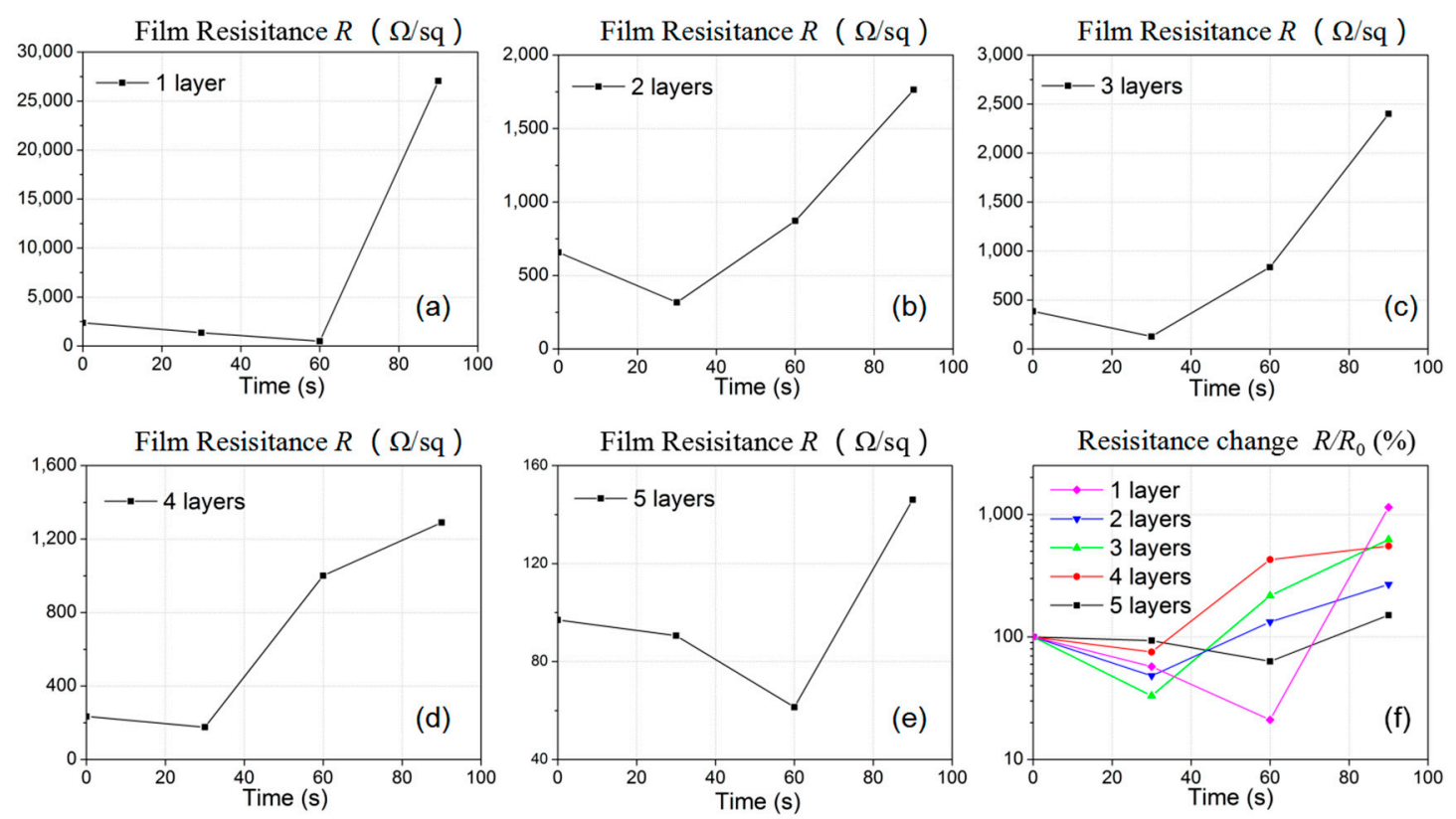

Figure 10. (a-e) Film resistance change with different time microwave heating with 1-5-layer AgNWs. (f) Relative resistance $R_{\mathrm{T}} / R_{0}$ with different time microwave heating with 1-5-layer AgNWs.

\subsection{AgNW Electrode for Glucose Concentration Sensing}

In this section, we coated the AgNWs on a thin film as working electrode for glucose concentration sensing application. We first tested the bio-compatibility of nanowire-coated PDMS film using T24 cells as described in Section 3.5. The cell growth conditions are shown in Figure 11. The cell number on the electrode grew stably and was found to be closed to that on the culture plate after $72 \mathrm{~h}$ growth. Therefore, the AgNWs are bio-compatible and suitable for the glucose concentration sensing.

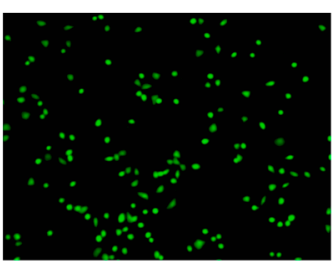

$12 \mathrm{~h}$

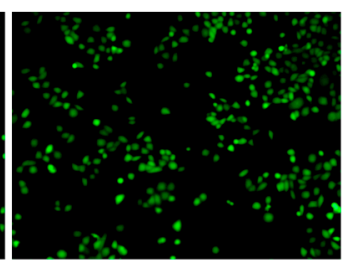

$24 \mathrm{~h}$

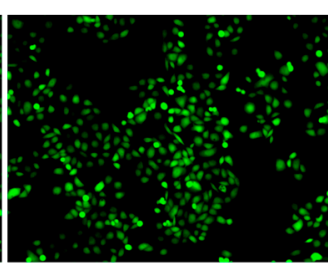

$48 \mathrm{~h}$

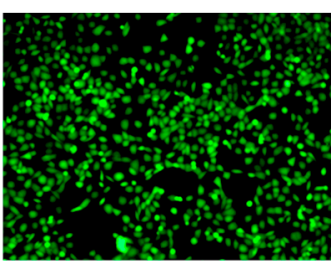

$72 \mathrm{~h}$

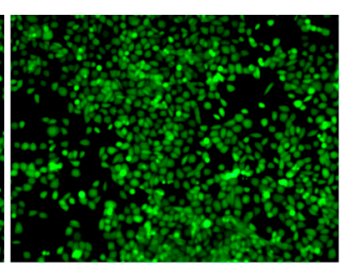

$72 \mathrm{~h}$ control

Figure 11. T24 cell growth on the AgNWs for $72 \mathrm{~h}$, and comparison with that on the culture plate.

Figure 12a,b shows the AgNW-coated PDMS electrode before and after coating the $\mathrm{GO}_{\mathrm{x}}$. As shown in Figure 12c, this electrode is soft and can be bent flexibly, therefore it has significant advantages in conformal biosensing applications. The AgNWs film was then immersed into glucose containing PBS solution as the working electrode for concentration sensing, as shown in Figure 13a. An electrical potential $V_{\text {glucose }}$ was applied between the working electrode and the counter electrode and the electric current from the working electrode to the reference electrode was measured. The cyclic voltammetry test was carried out by varying $V_{\text {glucose }}$ between $-0.2 \mathrm{~V}$ and $0.8 \mathrm{~V}$, as shown in Figure $13 \mathrm{~b}$. It can be seen that the reduction peaks occurred at $V_{\text {glucose }}=0.6 \mathrm{~V}$ in the cyclic voltammetry curve for different glucose concentrations. This voltage was set for the glucose concentration sensing. The glucose concentration was increased every $50 \mathrm{~s}$, and the measured electrical current increased correspondingly, as shown in Figure 13c. The average current at each concentration was plotted in Figure 13d, and followed a good linear relation with the glucose concentration between $1 \mathrm{mM}$ and $10 \mathrm{mM}$ regime. The slope of 1.13 and $R^{2}$ value of 
0.976 were obtained through linear fitting analysis, indicating a sensitivity of $1.13 \mu \mathrm{A} / \mathrm{mM}$ in this concentration regime. The electrical current values at glucose concentration of $16 \mathrm{mM}$ and $20 \mathrm{mM}$ were far below the fitting line, indicating that the sensitivity began to decrease in this high concentration regime.

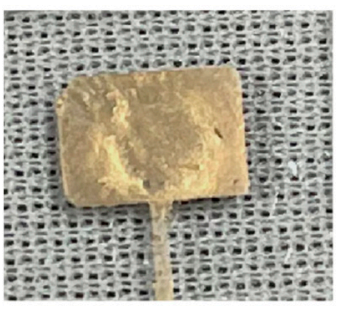

(a)

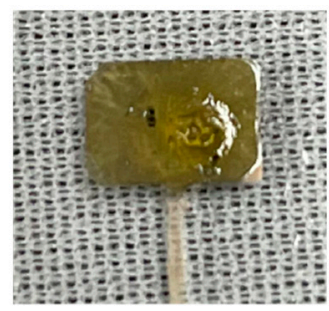

(b)

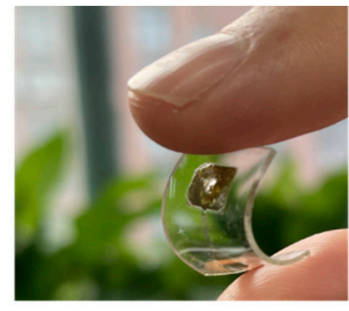

(c)

Figure 12. AgNWs electrode (a) before and (b) after $\mathrm{GO}_{\mathrm{x}}$ coating; (c) the bending of the electrode.
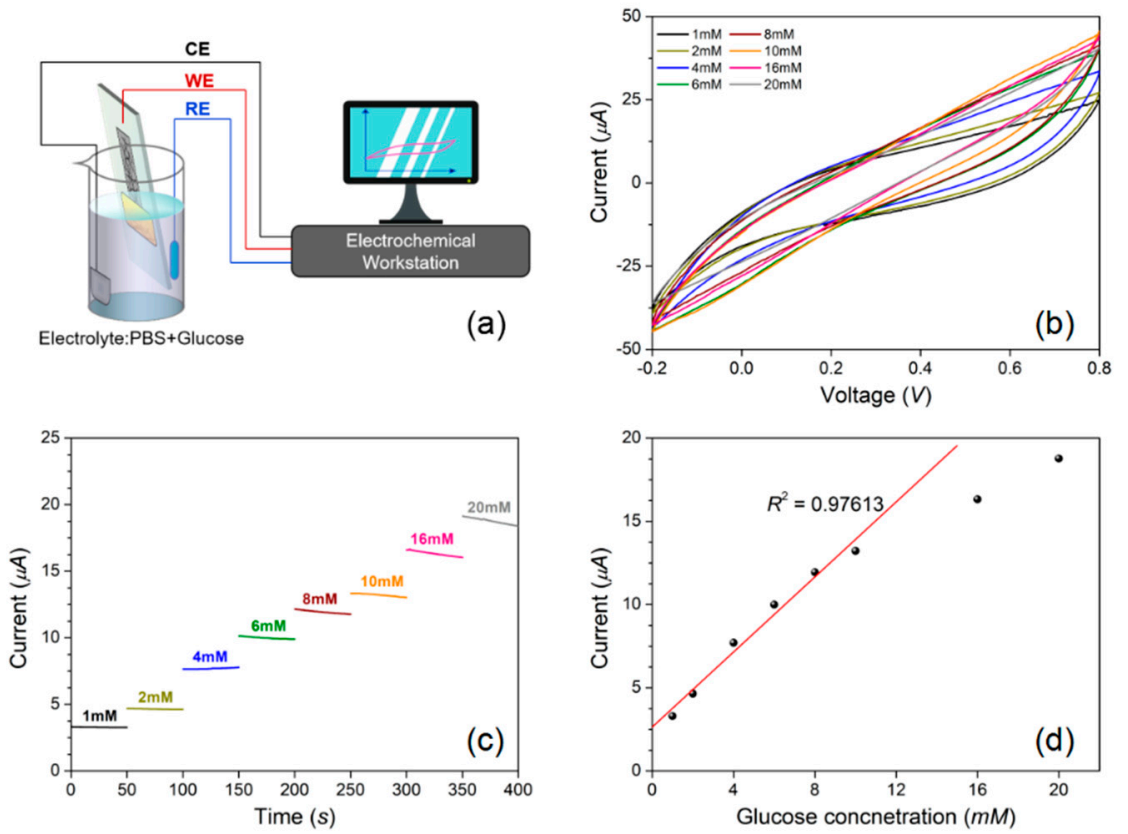

Figure 13. (a) Glucose sensing setup with the AgNW electrode; (b) cyclic voltammetry curve for the glucose sensing; (c) measured current for different glucose concentration; (d) average current at different glucose concentration and linear fitting.

\section{Conclusions}

In summary, we investigated the thermal effect of a microwave on Ag nanowires, and realized a significant conductivity enhancement on AgNW-coated thin film due to microwave welding. A soft and bio-compatible electrode based on the nanowire coating and microwave welding were demonstrated and was applied for glucose concentration sensing. The microwave heating on AgNWs was investigated by dispersing the nanowires in a nonpolar base solution. Linear relationships were obtained between the solution temperature increase and the nanowire weight concentration in the solution, nanowire diameter and applied microwave power. Nanowire welding was observed at the contact junctions under microwave radiation, and an electrical resistance reduction of $79 \%$ was achieved. Finally, we demonstrated a good bio-compatibility of the AgNW-based soft electrode, which realized a $1.13 \mu \mathrm{A} / \mathrm{mM}$ sensitivity for glucose sensing application. 
Author Contributions: Conceptualization, M.Z. and H.-J.C.; methodology, M.Z. and S.H.; validation, M.Z and S.H.; formal analysis, M.Z. and Z.-Y.X.; investigation, X.F. and S.H.; resources, H.-J.C.; data curation, W.Z.; writing-original draft preparation, M.Z.; writing-review and editing, W.Z.; visualization, X.L.; supervision, H.-J.C.; funding acquisition, M.Z. All authors have read and agreed to the published version of the manuscript.

Funding: The authors would like to acknowledge financial support from the China Postdoctoral Science Foundation (Grant No. 2020M683044, Grant No. 2020M683047), National Natural Science Foundation of China (Grant No. 61901535, Grant No. 61905046, Grant No. 61871003), Guangdong Basic and Applied Basic Research Foundation (Grant No. 2019A1515012087, 2020A1515110157, Grant No. 2021A1515011937), Science and Technology Program of Guangzhou (No. 202102080192, 202102020668), Provincial Key Laboratory of Information Photonics Technology (Guangdong University of Technology, Grant No. GKPT20-08).

Data Availability Statement: Raw data presented in this study are available on request from the corresponding author.

Conflicts of Interest: The authors declare no conflict of interest.

\section{References}

1. Tricoli, A.; Nasiri, N.; De, S. Wearable and Miniaturized Sensor Technologies for Personalized and Preventive Medicine. Adv. Funct. Mater. 2017, 27, 1605271. [CrossRef]

2. Hecht, D.S.; Hu, L.B.; Irvin, G. Emerging Transparent Electrodes Based on Thin Films of Carbon Nanotubes, Graphene, and Metallic Nanostructures. Adv. Mater. 2011, 23, 1482-1513. [CrossRef]

3. Quintero, A.V.; Molina-Lopez, F.; Smits, E.C.P.; Danesh, E.; van den Brand, J.; Persaud, K.; Oprea, A.; Barsan, N.; Weimar, U.; de Rooij, N.F.; et al. Smart RFID label with a printed multisensor platform for environmental monitoring. Flex. Print. Electron. 2016, 1, 025003. [CrossRef]

4. Falco, A.; Salmerón, J.F.; Loghin, F.C.; Lugli, P.; Rivadeneyra, A. Fully Printed Flexible Single-Chip RFID Tag with Light Detection Capabilities. Sensors 2017, 17, 534. [CrossRef]

5. Magliulo, M.; Mulla, M.Y.; Singh, M.; Macchia, E.; Tiwari, A.; Torsi, L.; Manoli, K. Printable and flexible electronics: From TFTs to bioelectronic devices. J. Mater. Chem. C 2015, 3, 12347-12363. [CrossRef]

6. Lu, Y.; Huang, J.Y.; Wang, C.; Sun, S.; Lou, J. Cold welding of ultrathin gold nanowires. Nat. Nanotechnol. 2010, 5, 218-224. [CrossRef]

7. Han, S.; Liu, C.; Xu, H.; Yao, D.; Yan, K.; Zheng, H.; Chen, H.-J.; Gui, X.; Chu, S.; Liu, C. Multiscale nanowire-microfluidic hybrid strain sensors with high sensitivity and stretchability. NPJ Flex. Electron. 2018, 2, 16. [CrossRef]

8. Beni, V.; Nilsson, D.; Arven, P.; Norberg, P.; Gustafsson, G.; Turner, A.P.F. Printed electrochemical instruments for biosensors. In Proceedings of the Symposium on Nano-Micro Sensors and Systems in Healthcare and Environmental Monitoring-227th ECS Meeting, Chicago, IL, USA, 24-28 May 2015; pp. 1-13.

9. Balachander, N.; Seshadri, I.; Mehta, R.J.; Schadler, L.S.; Borca-Tasciuc, T.; Keblinski, P.; Ramanath, G. Nanowire-filled polymer composites with ultrahigh thermal conductivity. Appl. Phys. Lett. 2013, 102, 093117. [CrossRef]

10. Lee, J.-Y.; Connor, S.T.; Cui, Y.; Peumans, P. Solution-Processed Metal Nanowire Mesh Transparent Electrodes. Nano Lett. 2008, 8, 689-692. [CrossRef]

11. De, S.; Higgins, T.M.; Lyons, P.E.; Doherty, E.M.; Nirmalraj, P.N.; Blau, W.J.; Boland, J.J.; Coleman, J.N. Silver Nanowire Networks as Flexible, Transparent, Conducting Films: Extremely High DC to Optical Conductivity Ratios. ACS Nano 2009, 3, 1767-1774. [CrossRef] [PubMed]

12. Mutiso, R.M.; Sherrott, M.C.; Rathmell, A.R.; Wiley, B.J.; Winey, K.I. Integrating Simulations and Experiments To Predict Sheet Resistance and Optical Transmittance in Nanowire Films for Transparent Conductors. ACS Nano 2013, 7, 7654-7663. [CrossRef]

13. Park, J.H.; Hwang, G.-T.; Kim, S.; Seo, J.; Park, H.-J.; Yu, K.; Kim, T.-S.; Lee, K.J. Flash-Induced Self-Limited Plasmonic Welding of Silver Nanowire Network for Transparent Flexible Energy Harvester. Adv. Mater. 2017, 29, 1603473. [CrossRef] [PubMed]

14. Garnett, E.C.; Cai, W.; Cha, J.J.; Mahmood, F.; Connor, S.T.; Greyson Christoforo, M.; Cui, Y.; McGehee, M.D.; Brongersma, M.L. Self-limited plasmonic welding of silver nanowire junctions. Nat. Mater. 2012, 11, 241-249. [CrossRef]

15. Park, J.H.; Han, S.; Kim, D.; You, B.K.; Joe, D.J.; Hong, S.; Seo, J.; Kwon, J.; Jeong, C.K.; Park, H.-J.; et al. Plasmonic-Tuned Flash Cu Nanowelding with Ultrafast Photochemical-Reducing and Interlocking on Flexible Plastics. Adv. Funct. Mater. 2017, $27,1701138$. [CrossRef]

16. Joe, D.J.; Kim, S.; Park, J.H.; Park, D.Y.; Lee, H.E.; Im, T.H.; Choi, I.; Ruoff, R.S.; Lee, K.J. Laser-Material Interactions for Flexible Applications. Adv. Mater. 2017, 29, 1606586. [CrossRef] [PubMed]

17. Tokuno, T.; Nogi, M.; Karakawa, M.; Jiu, J.; Nge, T.T.; Aso, Y.; Suganuma, K. Fabrication of silver nanowire transparent electrodes at room temperature. Nano Res. 2011, 4, 1215-1222. [CrossRef]

18. Langley, D.P.; Lagrange, M.; Giusti, G.; Jiménez, C.; Bréchet, Y.; Nguyen, N.D.; Bellet, D. Metallic nanowire networks: Effects of thermal annealing on electrical resistance. Nanoscale 2014, 6, 13535-13543. [CrossRef] 
19. Hosseinzadeh Khaligh, H.; Goldthorpe, I.A. Hot-rolling nanowire transparent electrodes for surface roughness minimization. Nanoscale Res. Lett. 2014, 9, 310. [CrossRef]

20. Xu, S.; Tian, M.; Wang, J.; Xu, J.; Redwing, J.M.; Chan, M.H.W. Nanometer-Scale Modification and Welding of Silicon and Metallic Nanowires with a High-Intensity Electron Beam. Small 2005, 1, 1221-1229. [CrossRef] [PubMed]

21. Zhang, K.; Li, J.; Fang, Y.; Luo, B.; Zhang, Y.; Li, Y.; Zhou, J.; Hu, B. Unraveling the solvent induced welding of silver nanowires for high performance flexible transparent electrodes. Nanoscale 2018, 10, 12981-12990. [CrossRef]

22. Yoon, S.-S.; Khang, D.-Y. Room-Temperature Chemical Welding and Sintering of Metallic Nanostructures by Capillary Condensation. Nano Lett. 2016, 16, 3550-3556. [CrossRef]

23. Dahal, N.; García, S.; Zhou, J.; Humphrey, S.M. Beneficial Effects of Microwave-Assisted Heating versus Conventional Heating in Noble Metal Nanoparticle Synthesis. ACS Nano 2012, 6, 9433-9446. [CrossRef] [PubMed]

24. Baghbanzadeh, M.; Carbone, L.; Cozzoli, P.D.; Kappe, C.O. Microwave-Assisted Synthesis of Colloidal Inorganic Nanocrystals. Angew. Chem. Int. Ed. 2011, 50, 11312-11359. [CrossRef] [PubMed]

25. Jung, P.-H.; Kim, Y.D.; Sung, Y.H.; Lee, H. Microwave welding of silver nanowires for highly transparent conductive electrodes. Phys. Status Solidi. 2017, 214, 1600908. [CrossRef]

26. Gedevanishvili, S.; Agrawal, D.; Roy, R. Microwave Combustion Synthesis and Sintering of Intermetallics and Alloys. J. Mater. Sci. Lett. 1999, 18, 665-668. [CrossRef]

27. Mishra, P.; Upadhyaya, A.; Sethi, G. Modeling of microwave heating of particulate metals. Metall. Mater. Trans. B 2006, 37, 839-845. [CrossRef]

28. Tohmyoh, H.; Fukui, S. Self-completed Joule heat welding of ultrathin Pt wires. Phys. Rev. B 2009, 80, 155403. [CrossRef] 